University of Nebraska - Lincoln

DigitalCommons@University of Nebraska - Lincoln

Faculty Publications from the Department of Engineering Mechanics

Mechanical \& Materials Engineering,

Department of

November 1995

\title{
Ultrasonic radiative transfer in polycrystalline media: Effects of a fluid-solid interface
}

Joseph A. Turner

University of Nebraska - Lincoln, jaturner@unl.edu

Richard L. Weaver

University of Illinois at Urbana-Champaign

Follow this and additional works at: https://digitalcommons.unl.edu/engineeringmechanicsfacpub

Part of the Mechanical Engineering Commons

Turner, Joseph A. and Weaver, Richard L., "Ultrasonic radiative transfer in polycrystalline media: Effects of a fluid-solid interface" (1995). Faculty Publications from the Department of Engineering Mechanics. 5. https://digitalcommons.unl.edu/engineeringmechanicsfacpub/5

This Article is brought to you for free and open access by the Mechanical \& Materials Engineering, Department of at DigitalCommons@University of Nebraska - Lincoln. It has been accepted for inclusion in Faculty Publications from the Department of Engineering Mechanics by an authorized administrator of DigitalCommons@University of Nebraska - Lincoln. 


\title{
Ultrasonic radiative transfer in polycrystalline media: Effects of a fluid-solid interface
}

\author{
Joseph A. Turner and Richard L. Weaver \\ Department of Theoretical and Applied Mechanics, 216 Talbot Laboratory, 104 South Wright Street, \\ University of Illinois at Urbana-Champaign, Urbana, Illinois 61801
}

(Received 14 November 1994; revised 9 May 1995; accepted 15 May 1995)

\begin{abstract}
In previous derivations of the ultrasonic radiative transfer equation (URTE) for the modeling of multiply scattered diffuse ultrasound in polycrystalline media, the boundary conditions appropriate to experiments performed in a water bath were not used. In the present work, this fluid-solid boundary condition is discussed as it applies to the URTE. An interpolation scheme is developed that is consistent with the discrete ordinates method used for the solution of the URTE. Steady-state and time-dependent results are presented for the solution of the URTE with a fluid-solid boundary condition. The steady-state results show that diffusive behavior is exhibited nearer the specimen surface than before. The time-dependent results show the qualitative change one might expect from such a boundary condition. (C) 1995 Acoustical Society of America.
\end{abstract}

PACS numbers: 43.20.Bi, 43.20.Gp, 43.35.Cg

\section{INTRODUCTION}

Microstructural characterization of polycrystalline metals is often performed using ultrasonic backscatter techniques. $^{1-3}$ The backscattered diffuse or incoherent signals, also called grain noise, contain microstructural information about grain size, orientation, and composition which is useful for materials characterization. The grain noise can also interfere with flaw detection. Understanding the scattering mechanism is thus important. When the time and/or length scales of a backscatter experiment are long compared with the time and length scales of the random scattering events occurring within the medium, multiple-scattering effects become important. The multiple-scattering problem has two limits. In the limit of early times or weakly scattering materials, and for experiments involving focused transducers, a single-scattering approximation has been successful for modeling grain noise. ${ }^{1-3}$ This assumption implies that the incident wave strikes only one scatterer before being detected. In the opposite limit, at late times after the energy has scattered many times, the behavior is governed by a diffusion equation. ${ }^{4,5}$ The intermediate multiple-scattering regime has not, however, been fully utilized for microstructural characterization possibly because of the lack of an adequate theory with which to describe corresponding experiments.

A method was recently proposed to model the multiple scattering of diffuse ultrasound in polycrystalline materials. ${ }^{5-7}$ It has its foundations in optical radiative transfer theory which was developed to quantify the diffuse scattering of light from planetary and stellar atmospheres. ${ }^{8-10}$ The ultrasonic radiative transfer equation (URTE) is derived for a polycrystalline medium through an examination of ensemble averaged responses of the elastic wave equation by use of the Bethe-Salpeter equation. ${ }^{5,6}$ The URTE is expected to be valid within the limit of its primary assumption that the material heterogeneity is weak. Many materials of interest satisfy this requirement and thus are expected to be modeled appropriately by the URTE.
The URTE was previously solved for both the steadystate and time-dependent cases. ${ }^{6,7}$ These results showed that the multiply scattered energy contains much more microstructural information than is available to conventional singly scattered measurements. ${ }^{1-3}$ For both of these problems a transparent boundary condition was assumed such that the diffuse energy entering the medium was set to zero. However, experiments with diffuse ultrasound are ordinarily performed in a water bath. In this case, a large amount of the energy incident on the front face of the specimen will be coherently reflected and not enter the specimen. A considerable amount of the internally scattered diffuse energy will likewise reflect from the interface back into the specimen and be further scattered. Because these effects may become important after only a few scatterings, they must be appropriately included in the ultrasonic radiative transfer model. Reflective boundary conditions are also important when the specimen cannot be accurately modeled as semi-infinite so that reflections from the lower boundary must also be included.

In the next section the URTE with appropriate fluidsolid boundary conditions is briefly discussed. Section II contains the derivation of the reflection and transmission coefficients necessary for the reflective boundary condition. Section III contains the development of an interpolation scheme which is necessary for use with the discrete ordinates solution method. ${ }^{11}$ Steady-state and time-dependent results of the URTE with a reflective boundary condition are then presented in Secs. IV and V, respectively.

\section{ULTRASONIC RADIATIVE TRANSFER THEORY}

Consider a polycrystalline specimen with Voigt average longitudinal and transverse wave speeds $c_{L}$ and $c_{T}$, immersed in a fluid bath with wave speed $c_{f}$. The fourth-rank elastic moduli tensor is assumed to be of the form

$$
C_{i j k l}(\mathbf{x})=C_{i j k l}^{0}+\gamma_{i j k l}(\mathbf{x}),
$$




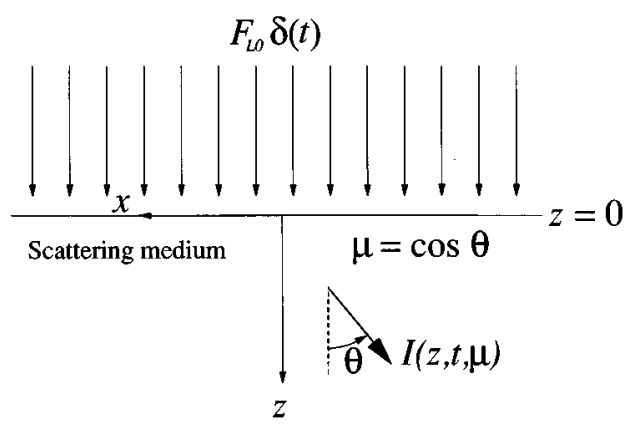

FIG. 1. Geometry of the problem.

where $C_{i j k l}^{0}$ are the Voigt average moduli and $\gamma_{i j k l}$ are the elastic moduli fluctuations which depend upon the position vector $\mathbf{x}$. The fluctuations are assumed small such that $\boldsymbol{\gamma} \ll \mathbf{C}^{0}$ and have an ensemble average of zero $(\langle\gamma\rangle=0)$. The material is presumed statistically homogeneous and isotropic which implies that $\mathbf{C}^{0}$ is independent of $\mathbf{x}$ and of the standard isotropic form. ${ }^{5,6}$ The material property of interest is then the covariance of the moduli fluctuations, $\langle\gamma \gamma\rangle$, which is assumed to be of the form

$$
\left\langle\gamma_{\alpha \beta \gamma \delta}(\mathbf{x}) \gamma_{i j k l}\left(\mathbf{x}^{\prime}\right)\right\rangle=\Xi_{i j k l}^{\alpha \beta \gamma \delta} W\left(\left|\mathbf{x}-\mathbf{x}^{\prime}\right|\right) .
$$

The eighth-rank tensor $\Xi$ is assumed independent of position. The geometric correlation function $W$ defines the probability that two points within the medium, $\mathbf{x}$ and $\mathbf{x}^{\prime}$, lie within the same crystallite. The form of Eq. (2) is obtained from the assumption that all crystallite orientations are equally likely and that the material is statistically isotropic and statistically homogeneous. ${ }^{5,6}$

The specimen is excited by a normally incident plane wave with incident flux $F_{L 0}$ as shown in Fig. 1. Furthermore, for the time-dependent problem, the incident field is idealized as a delta function in time as discerned on the time scale of the slowly evolving diffuse field. One may think of this field as a short tone burst with center frequency $\omega$. Time domain results for longer tone bursts may be found by convolution.

The URTE for this problem is ${ }^{6,7}$

$$
\begin{aligned}
\mu \frac{\partial \underline{I}(z, t, \mu)}{\partial z}+\underline{\underline{c}}^{-1} \frac{\partial \underline{I}(z, t, \mu)}{\partial t}+(\underline{\underline{\kappa}}+\underline{\underline{v}}) \underline{I}(z, t, \mu) \\
=\frac{1}{2} \int_{-1}^{+1} \underline{\underline{P}}\left(\mu ; \mu^{\prime}\right) \underline{I}\left(z, t, \mu^{\prime}\right) d \mu^{\prime}+\underline{S}_{L}\left(\mu, \mu_{0}\right) \\
\quad \times e^{-\sigma_{L} z / \mu_{0}} \delta\left(t-z / c_{L} \mu_{0}\right),
\end{aligned}
$$

where the Stokes vector $\underline{I}$ contains the five elastic Stokes parameters, one longitudinal, $I_{L}$, and four shear, $I_{S V}, I_{S H}$, $U$, and $V$, which characterize the diffuse intensity. The specific intensities $I_{L}, I_{S V}$, and $I_{S H}$ are proportional to the respective average square longitudinal, shear vertical, and shear horizontal displacements and have units of energy per unit area per unit time per unit solid angle. The parameters $U$ and $V$ are related to the coherent interference between the two shear waves which is maintained over long distances because of the identical wave speeds of these waves. The
Stokes vector is a function of depth, time, and the direction of propagation defined by $\mu=\cos \theta$, where $\theta$ is the angle between the $z$ axis and the direction of propagation. The matrices $\underline{\underline{c}}, \underline{\underline{\kappa}}$, and $\underline{\underline{v}}$ define the wave speed, scattering, and absorption matrices. ${ }^{6}$

The Mueller matrix $\stackrel{\underline{P}}{\text { governs }}$ the scattering between the Stokes parameters which comprise the multiplescattering process. Each component $P_{i j}$ is of the form

$$
P_{i j} \propto \tilde{W} \Xi_{\ldots . . \hat{\mathbf{u}} \hat{\mathbf{p}} \hat{\mathbf{p}} \hat{\mathbf{s}} \hat{\mathbf{r}}},
$$

where $\hat{\mathbf{u}}, \hat{\mathbf{v}}, \hat{\mathbf{q}}$, and $\hat{\mathbf{r}}$ are the displacement vectors and $\hat{\mathbf{p}}$ and $\hat{\mathbf{s}}$ are the incident and scatter directions which are separated by an angle $\Theta_{p s}$. Thus $\underline{\underline{P}}$ contains combinations of inner products of the covariance of elastic moduli fluctuations and wave vectors. The matrix $\underline{\underline{P}}$ is also a function of the spatial Fourier transform of the two-point geometric correlation function of the material properties given by $\tilde{W}$, which is a function of $\Theta_{p s}$ and the length scale of the medium. It has been derived for polycrystalline aggregates of cubic and hexagonal crystallites and is parametrically dependent upon the excitation frequency $\omega$. The Mueller matrix contains all of the relevant statistical information about the scattering medium.

The URTE is a first-order integro-partial differential equation which, for a general Mueller matrix, has nontrivial solutions. The left-hand side of the URTE represents the propagation and subsequent attenuation (due to both scattering and absorption) of the Stokes parameters in the $\mu$ direction. The right-hand side is composed of two source terms. The integral term represents the total energy entering the scattering volume from the $\mu^{\prime}$ direction that scatters into the direction $\mu$. Thus this term appears as a secondary source of intensity. The second term on the right-hand side of Eq. (3) containing $\underline{S}_{L}$ is the source due to the incident wave that has singly scattered. The quantity $\underline{S}_{L}$ is proportional to the flux of the incident wave. Numerical solutions for both the steadystate and the time-dependent URTE have been developed using the discrete ordinates method. ${ }^{6,711}$ For both of these problems a transparent boundary condition was assumed such that the diffuse energy entering the medium at the surface was set to zero, $\underline{I}(z=0, t, \mu>0)=0$. This boundary condition does not appropriately model experiments performed in a water bath. Therefore a modification is necessary. Perhaps the simplest way to include the surface reflection is to modify this homogeneous boundary condition. With a reflective boundary, the downward intensity is linearly related to the upward intensity as follows:

$$
\underline{I}(z=0, t, \mu>0)=\underline{\underline{R}} \underline{I}(z=0, t, \mu<0) .
$$

The reflection matrix $\stackrel{R}{=}$ relates the upward propagating $(\mu<0)$ Stokes parameters to the downward propagating $(\mu>0)$ Stokes parameters at the specimen surface, $z=0$. Once the intensities in the solid are calculated, the intensity in the fluid is found using the transmission vector $\underline{T}$ which relates the Stokes parameters in the solid to the fluid Stokes parameter $I_{f}$ by

$$
I_{f}=\underline{T}^{T} \underline{I}(z=0, t, \mu<0),
$$

where the $T$ superscript denotes the transpose. 


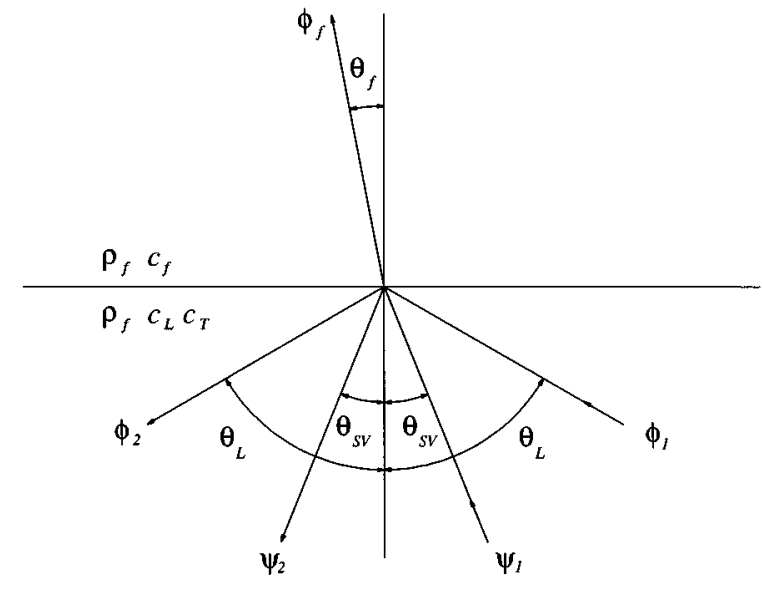

FIG. 2. Reflection and transmission of potential functions.

The components of $\underline{\underline{R}}$ and $\underline{T}$ are related to the power reflection coefficients prevalent in the literature. ${ }^{12-15}$ These components are now derived.

\section{DERIVATION OF THE REFLECTION MATRIX}

The form of the reflection matrix and transmission vector given in Eqs. (5) and (6) may immediately be written as

$$
\begin{aligned}
& \underline{\underline{R}}=\left[\begin{array}{ccccc}
R_{L L}^{I} & R_{S V L}^{I} & 0 & 0 & 0 \\
R_{L S V}^{I} & R_{S V S V}^{I} & 0 & 0 & 0 \\
0 & 0 & 1 & 0 & 0 \\
0 & 0 & 0 & R_{U U}^{I} & R_{V U}^{I} \\
0 & 0 & 0 & R_{U V}^{I} & R_{V V}^{I}
\end{array}\right], \\
& \underline{T}=\left\{\begin{array}{c}
T_{L f}^{I} \\
T_{S V f}^{I} \\
0 \\
0 \\
0
\end{array}\right\} .
\end{aligned}
$$

The superscript $I$ is used as a reminder that these are reflection and transmission coefficients for the specific intensities. The longitudinal and shear vertical Stokes parameters couple to each other and are the only intensities that couple with the fluid. The shear horizontal intensity reflects entirely into itself. The $U$ and $V$ components affect only each other as discussed by Tsang et al. ${ }^{10}$ for the electromagnetic case. Because $U$ and $V$ describe the coherence of the two shear components, ${ }^{6,11}$ these two parameters do not transmit into the fluid because of the zero transmission of the shear horizontal mode.

The derivation of the components of $R$ and $\underline{T}$ is now discussed. The reflection and refraction of incident longitudinal and shear waves is given most compactly by Brekhovskikh and Godin ${ }^{15}$ in terms of potential functions. Their work will be outlined here.

Consider the reflection and transmission at an interface shown in Fig. 2 for a fluid-solid system. The upper halfspace is a fluid with density $\rho_{f}$ and wave speed $c_{f}$. The lower half-space is the scattering solid with density $\rho$ and longitudinal and transverse wave speeds $c_{L}$ and $c_{T}$, respec- tively. An incident potential, $\phi_{1}$ or $\psi_{1}$, reflects into $\phi_{2}$ and $\psi_{2}$ and reflects into $\phi_{f}$ in the fluid. Since the fluid does not support shear waves, $\psi_{f}=0$. The longitudinal and shear vertical displacement amplitudes are related to the potentials as $u_{L}=\omega \phi / c_{L}$ and $u_{S V}=\omega \psi / c_{T}$, respectively. The pressure in the fluid is $p=\rho \omega^{2} \phi_{f}$. For an incident longitudinal plane wave described by the potential $\phi_{1}$, the reflected and refracted potentials are proportional to the incident potential and given by

$$
\phi_{2}=V_{L L} \phi_{1}, \quad \psi_{2}=V_{L S V} \phi_{1}, \quad \phi_{f}=W_{L f} \phi_{1} .
$$

For an incident shear vertical wave described by the potential $\psi_{1}$, the reflected and refracted potentials are given by

$$
\phi_{2}=V_{S V L} \psi_{1}, \quad \psi_{2}=V_{S V S V} \psi_{1}, \quad \phi_{f}=W_{S V f} \psi_{1} .
$$

The quantities $V_{L L}, V_{L S V}, W_{L f}, V_{S V L}, V_{S V S V}$, and $W_{S V f}$ given in Eqs. (8) and (9) are the reflection and transmission coefficients for the potentials defined as ${ }^{15}$

$$
\begin{aligned}
& V_{L L}=\frac{Z_{f}+Z_{S V} \sin ^{2} 2 \theta_{S V}-Z_{L} \cos ^{2} 2 \theta_{S V}}{Z_{f}+Z_{S V} \sin ^{2} 2 \theta_{S V}+Z_{L} \cos ^{2} 2 \theta_{S V}}, \\
& V_{L S V}=-\frac{2\left(1-V_{L L}\right) \cot \theta_{L} \sin ^{2} \theta_{S V}}{\cos 2 \theta_{S V}}, \\
& W_{L f}=\frac{\left(1-V_{L L}\right) \tan \theta_{f} \cot \theta_{L}}{\cos 2 \theta_{S V}},
\end{aligned}
$$

for Eqs. (8) and

$$
\begin{aligned}
& V_{S V S V}=-\frac{Z_{f}+Z_{L} \cos ^{2} 2 \theta_{S V}-Z_{S V} \sin ^{2} 2 \theta_{S V}}{Z_{f}+Z_{L} \cos ^{2} 2 \theta_{S V}+Z_{S V} \sin ^{2} 2 \theta_{S V}}, \\
& V_{S V L}=\frac{\left(1+V_{S V S V}\right) \tan \theta_{L} \cos 2 \theta_{S V}}{2 \sin ^{2} \theta_{S V}}, \\
& W_{S V f}=\frac{\left(1+V_{S V S V}\right) \tan \theta_{f}}{2 \sin ^{2} \theta_{S V}},
\end{aligned}
$$

for Eqs. (9) where the impedances are defined as

$$
Z_{f}=\frac{\rho_{f} c_{f}}{\cos \theta_{f}}, \quad Z_{L}=\frac{\rho c_{L}}{\cos \theta_{L}}, \quad Z_{S V}=\frac{\rho c_{T}}{\cos \theta_{S V}} .
$$

At the interface between the fluid and the solid, energy flux normal to the boundary must balance. For an incident longitudinal wave this energy balance is given by ${ }^{14,15}$

$$
\begin{aligned}
1 & =\frac{E_{L}^{+}}{E_{L}^{-}}+\frac{E_{S V}^{+}}{E_{L}^{-}}+\frac{E_{f}^{-}}{E_{L}^{-}} \\
& =\left|V_{L L}\right|^{2}+\frac{\tan \theta_{L}}{\tan \theta_{S V}^{+}}\left|V_{L S V}\right|^{2}+\frac{\rho_{f} \tan \theta_{L}}{\rho \tan \theta_{f}}\left|W_{L f}\right|^{2} .
\end{aligned}
$$

For an incident $S V$ wave

$$
\begin{aligned}
1 & =\frac{E_{S V}^{+}}{E_{S V}^{-}}+\frac{E_{L}^{+}}{E_{S V}^{-}}+\frac{E_{f}^{-}}{E_{S V}^{-}} \\
& =\left|V_{S V S V}\right|^{2}+\frac{\tan \theta_{S V}}{\tan \theta_{L}^{+}}\left|V_{S V L}\right|^{2}+\frac{\rho_{f} \tan \theta_{S V}}{\rho \tan \theta_{f}}\left|W_{S V f}\right|^{2} .
\end{aligned}
$$




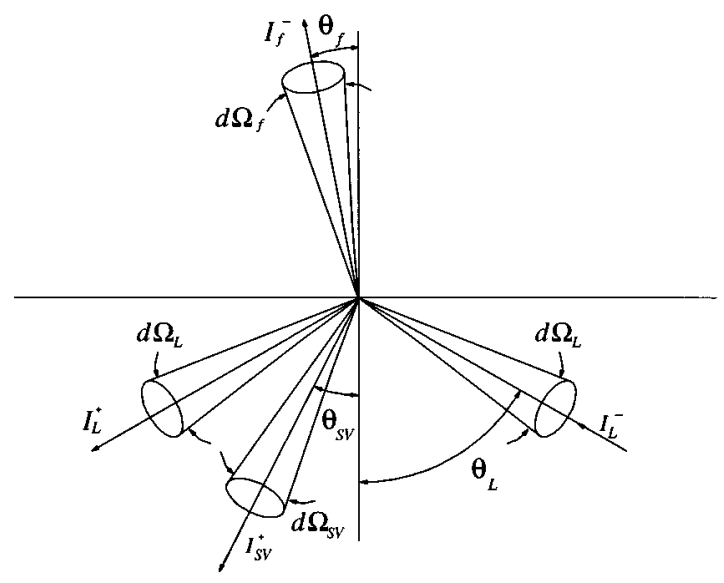

FIG. 3. Reflection and transmission of Stokes parameters.

In Eqs. (13) and (14) the + and - superscripts designate energy propagating in the $\mu>0$ and $\mu<0$ directions, respectively. From these two equations the power reflection and transmission coefficients may be defined as

$$
\begin{aligned}
& R_{L L}^{\pi}=\left|V_{L L}\right|^{2}, \quad R_{L S V}^{\pi}=\left|V_{L S V}\right|^{2} \frac{\tan \theta_{L}}{\tan \theta_{S V}}, \\
& T_{L f}^{\pi}=\left|W_{L f}\right|^{2} \frac{\rho_{f} \tan \theta_{L}}{\rho \tan \theta_{f}}, \\
& R_{S V S V}^{\pi}=\left|V_{S V S V}\right|^{2}, \quad R_{S V L}^{\pi}=\left|V_{S V L}\right|^{2} \frac{\tan \theta_{S V}}{\tan \theta_{L}}, \\
& T_{S V f}^{\pi}=\left|W_{S V f}\right|^{2} \frac{\rho_{f} \tan \theta_{S V}}{\rho \tan \theta_{f}},
\end{aligned}
$$

where the superscript $\pi$ implies that these are power reflection and transmission coefficients. With these definitions, the flux balance equations become $R_{L L}^{\pi}+R_{L S V}^{\pi}+T_{L f}^{\pi}=1$ and $R_{S V S V}^{\pi}+R_{S V L}^{\pi}+T_{S V f}^{\pi}=1$. Ergin ${ }^{16}$ has plotted the square root of these power coefficients as a function of incident angle for three different parameter regimes.

The Stokes parameters that reflect and transmit must also satisfy this flux balance. These parameters were defined as the energy per time per area per solid angle. Thus any beam divergence due to reflection or transmission must be examined. The reflection and transmission of an incident longitudinal Stokes parameter are depicted in Fig. 3. A longitudinal Stokes parameter $I_{L}^{-}$with beam width $d \Omega_{L}$ impinges at the interface at orientation angle $\theta_{L}$, defined from the normal. Two intensities are reflected from the interface: a longitudinal intensity $I_{L}^{+}$with beamwidth $d \Omega_{L}$ and orientation angle $\theta_{L}$ and a shear vertical intensity $I_{S V}^{+}$with beamwidth $d \Omega_{S V}$ and orientation angle $\theta_{S V}$. One intensity, $I_{f}$, is transmitted into the fluid with beamwidth $d \Omega_{f}$ and orientation angle $\theta_{f}$. The incremental beamwidth $d \Omega$ is related to the orientation angle $\theta$ and azimuthal angle $\phi$ by $d \Omega$ $=\sin \theta d \theta d \phi$ for each of the particular beams.

Conservation of power flux at the surface implies that for an incident longitudinal Stokes parameter
$I_{L}^{-} \cos \theta_{L} d a d \Omega_{L}=I_{L}^{+} \cos \theta_{L} d a d \Omega_{L}$

$$
\begin{aligned}
& +I_{S V}^{+} \cos \theta_{S V} d a d \Omega_{S V} \\
& +I_{f}^{-} \cos \theta_{f} d a d \Omega_{f}
\end{aligned}
$$

or

$$
1=\frac{I_{L}^{+}}{I_{L}^{-}}+\frac{I_{S V}^{+}}{I_{L}^{-}} \frac{\cos \theta_{S V} d \Omega_{S V}}{\cos \theta_{L} d \Omega_{L}}+\frac{I_{f}^{-}}{I_{L}^{-}} \frac{\cos \theta_{f} d \Omega_{f}}{\cos \theta_{L} d \Omega_{L}} .
$$

Similarly, for an incident shear vertical intensity

$$
1=\frac{I_{S V}^{+}}{I_{S V}^{-}}+\frac{I_{L}^{+}}{I_{S V}^{-}} \frac{\cos \theta_{L} d \Omega_{L}}{\cos \theta_{S V} d \Omega_{S V}}+\frac{I_{f}^{-}}{I_{S V}^{-}} \frac{\cos \theta_{f} d \Omega_{f}}{\cos \theta_{S V} d \Omega_{S V}} .
$$

These equations may be simplified by differentiating Snell's Law,

$$
\frac{\sin \theta_{L}}{c_{L}}=\frac{\sin \theta_{S V}}{c_{T}}=\frac{\sin \theta_{f}}{c_{f}},
$$

which gives

$$
\frac{\cos \theta_{L} d \theta_{L}}{c_{L}}=\frac{\cos \theta_{S V} d \theta_{S V}}{c_{T}}=\frac{\cos \theta_{f} d \theta_{f}}{c_{f}} .
$$

Multiplying Eqs. (19) and (20) together and noting that $d \phi_{L}=d \phi_{S V}=d \phi_{f}$ gives the relation between the solid angles needed in Eqs. (17) and (18):

$$
\frac{\cos \theta_{L} d \Omega_{L}}{c_{L}^{2}}=\frac{\cos \theta_{S V} d \Omega_{S V}}{c_{T}^{2}}=\frac{\cos \theta_{f} d \Omega_{f}}{c_{f}^{2}} .
$$

The power flux relationship that the Stokes parameters must satisfy is then

$$
1=\frac{I_{L}^{+}}{I_{L}^{-}}+\frac{I_{S V}^{+}}{I_{L}^{-}} \frac{c_{T}^{2}}{c_{L}^{2}}+\frac{I_{f}^{-}}{I_{L}^{-}} \frac{c_{f}^{2}}{c_{L}^{2}}
$$

for an incident longitudinal wave and

$$
1=\frac{I_{S V}^{+}}{I_{S V}^{-}}+\frac{I_{L}^{+}}{I_{S V}^{-}} \frac{c_{L}^{2}}{c_{T}^{2}}+\frac{I_{f}^{-}}{I_{S V}^{-}} \frac{c_{f}^{2}}{c_{T}^{2}}
$$

for an incident shear vertical wave. Thus a beam divergence of the Stokes parameters is seen which is proportional to the square of the wave-speed ratio for the respective Stokes parameters. Comparing Eqs. (13) and (14) for the energy flux balance with Eqs. (22) and (23) for the Stokes parameter flux balance leads to the definition of the specific intensity reflection and transmission coefficients. For an incident longitudinal Stokes parameter

$$
\begin{aligned}
& R_{L L}^{I}=R_{L L}^{\pi}=\left|V_{L L}\right|^{2}, \\
& R_{L S V}^{I}=R_{L S V}^{\pi} \frac{c_{L}^{2}}{c_{T}^{2}}=\left|V_{L S V}\right|^{2} \frac{\tan \theta_{L}}{\tan \theta_{S V}} \frac{c_{L}^{2}}{c_{T}^{2}}, \\
& T_{L f}^{I}=T_{L f}^{\pi} \frac{c_{L}^{2}}{c_{f}^{2}}=\left|W_{L f}\right|^{2} \frac{\rho_{f} \tan \theta_{L}}{\rho \tan \theta_{f}} \frac{c_{L}^{2}}{c_{f}^{2}},
\end{aligned}
$$

and for an incident shear vertical 


$$
\begin{aligned}
& R_{S V S V}^{I}=R_{S V S V}^{\pi}=\left|V_{S V S V}\right|^{2}, \\
& R_{S V L}^{I}=R_{S V L}^{\pi} \frac{c_{T}^{2}}{c_{L}^{2}}=\left|V_{S V L}\right|^{2} \frac{\tan \theta_{S V}}{\tan \theta_{L}} \frac{c_{T}^{2}}{c_{L}^{2}}, \\
& T_{S V f}^{I}=T_{S V f}^{\pi} \frac{c_{T}^{2}}{c_{f}^{2}}=\left|W_{S V f}\right|^{2} \frac{\rho_{f} \tan \theta_{S V}}{\rho \tan \theta_{f}} \frac{c_{T}^{2}}{c_{f}^{2}} .
\end{aligned}
$$

The $U$ and $V$ reflection coefficients are related to the two shear component reflections. The definitions of $U$ and $V$ and the fact that the shear horizontal mode reflects completely gives the reflection coefficients for $U$ and $V$,

$$
\begin{aligned}
& R_{U U}^{I}=R_{V V}^{I}=-\operatorname{Re}\left(V_{S V S V}\right), \\
& R_{U V}^{I}=-R_{V U}^{I}=-\operatorname{Im}\left(V_{S V S V}\right) .
\end{aligned}
$$

These coefficients agree with those given by Tsang et al. ${ }^{10}$ for the reflection of electromagnetic waves from the interface between two materials with differing permittivities.

The derived reflection and transmission coefficients are now discussed with regard to implementation using the discrete ordinates method which is used to solve Eq. (3).

\section{IMPLEMENTATION USING DISCRETE ORDINATES}

The solution method outlined previously ${ }^{11}$ made use of the discrete ordinates method in which the intensities were discretized in angle. One may immediately see a problem that arises when this method is used with the reflection boundary condition. When a longitudinal (or shear vertical) intensity is incident on the interface, the portion that mode converts into shear vertical (or longitudinal) will not, in general, fall on the chosen ordinates. This complication would arise in electromagnetic problems only when the interface of two dissimilar materials is considered. For the ultrasonic case, some type of interpolation is then necessary. An interpolation scheme is derived here that is consistent with the underlying character of the discrete ordinates method.

According to the methodology of the discrete ordinates method, ${ }^{8-10}$ the integral of some function $f(\mu)$ is approximated using Gaussian quadrature as

$$
\begin{aligned}
& \int_{-1}^{+1} f(\mu) d \mu \cong \sum_{j=-N}^{+N} a_{j} f\left(\mu_{j}\right) \\
& a_{j}=\frac{1}{P_{2 N}^{\prime}\left(\mu_{j}\right)} \int_{-1}^{+1} \frac{P_{2 N}(\mu)}{\left(\mu-\mu_{j}\right)} d \mu,
\end{aligned}
$$

where $P_{2 N}$ is the $2 N$ th Legendre polynomial with the prime denoting a derivative. The $a_{j}$ 's are the quadrature weights and the $\mu_{j}$ 's the quadrature divisions which are the zeroes of $P_{2 N}$. This approximation is equivalent to expanding the integrand in terms of polynomial basis functions:

$$
\begin{aligned}
f(\mu) & \cong \sum_{j=-N}^{+N} f\left(\mu_{j}\right) \frac{P_{2 N}(\mu)}{\left(\mu-\mu_{j}\right) P_{2 N}^{\prime}\left(\mu_{j}\right)} \\
& =\sum_{j=-N}^{+N} f\left(\mu_{j}\right) \varphi_{j}(\mu),
\end{aligned}
$$

where the expansion polynomials $\varphi_{j}(\mu)$ defined in Eq. (28) equal unity when $\mu=\mu_{j}$ and equal zero when $\mu=\mu_{i \neq j}$. Thus the approximation gives $f(\mu)$ exactly at each of the ordinates and is exact for all $\mu$ if $f(\mu)$ is a polynomial of order $2 N-1$ or less. ${ }^{9}$ This expansion, Eq. (28), can be used to approximate the intensity at some off-ordinate value given the values on-ordinate. The value of $I$ in a direction defined by $\mu_{i}$ off-ordinate is approximated by

$$
I\left(\mu_{i}\right) \cong \sum_{j=-N}^{+N} I\left(\mu_{j}\right) \frac{P_{2 N}\left(\mu_{i}\right)}{\left(\mu_{i}-\mu_{j}\right) P_{2 N}^{\prime}\left(\mu_{j}\right)} .
$$

Equation (29) implies that the off-ordinate intensity is related to each of the on-ordinate intensities. The interpolation given by Eq. (29) will be exact if the intensity is well defined by a $2 N-1$ degree polynomial. Thus the error associated with this interpolation is related to the higher-order behavior of the intensities.

The problem of mode conversion at the surface may now be examined. An on-ordinate downward $I_{S V}$ at the surface is related to some off-ordinate upward $I_{L}$ through the reflection coefficient

$$
I_{S V}\left(\mu_{k}>0\right)=R_{L S V}^{I} I_{L}\left(\mu_{i}<0\right),
$$

where $\mu_{k}$ is on-ordinate and $\mu_{i}$ is off-ordinate. The two angles which define $\mu_{k}$ and $\mu_{i}$ are related through Snell's law, Eq. (19). Using the approximation given in Eq. (29), Eq. (30) becomes

$$
I_{S V}\left(\mu_{k}>0\right)=R_{L S V}^{I} \sum_{j=-N}^{-1} I_{L}\left(\mu_{j}<0\right) \frac{P_{2 N}\left(\mu_{i}\right)}{\left(\mu_{i}-\mu_{j}\right) P_{2 N}^{\prime}\left(\mu_{j}\right)} .
$$

This approximation provides a relation between the onordinate downward $S V$ intensity in terms of the on-ordinate upward $L$ intensities. A similar approximation is made for the $S V$ to $L$ conversion:

$$
I_{L}\left(\mu_{k}>0\right)=R_{S V L}^{I} \sum_{j=-N}^{-1} I_{S V}\left(\mu_{j}<0\right) \frac{P_{2 N}\left(\mu_{i}\right)}{\left(\mu_{i}-\mu_{j}\right) P_{2 N}^{\prime}\left(\mu_{j}\right)} .
$$

In the previous numerical discussion, ${ }^{6,7,11}$ a vector I was defined as

$$
\mathbf{I}(z)=\left\{\begin{array}{l}
\mathbf{I}^{-}(z) \\
\mathbf{I}^{+}(z)
\end{array}\right\}=\left\{\begin{array}{c}
\underline{I}\left(z, \mu_{-N}\right) \\
\cdot \\
\cdot \\
\cdot \\
\underline{I}\left(z, \mu_{+N}\right)
\end{array}\right\},
$$

which contains the Stokes parameters for each of the directional components. The previously used transparent boundary condition implied that $\mathbf{I}(z=0)=0$. The reflective boundary condition for this vector is now

$$
\mathbf{I}^{+}(z=0)=\mathbf{R I}^{-}(z=0),
$$

where the discretized reflection matrix $\mathbf{R}$ has the form 


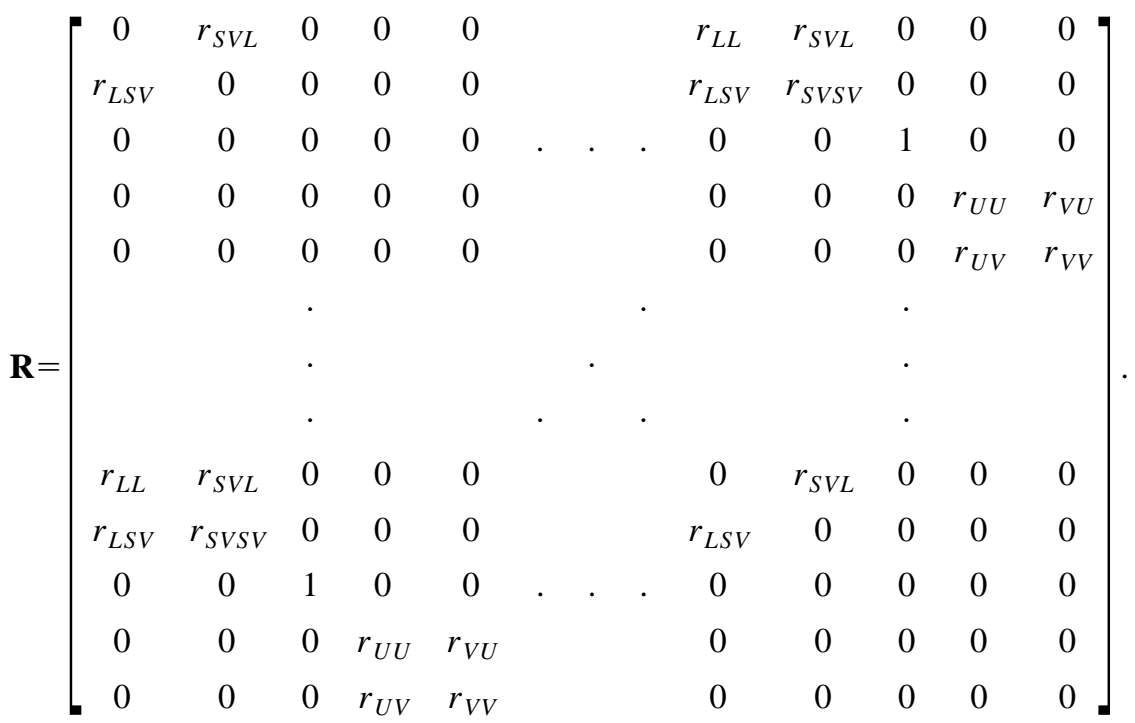

The components of $\mathbf{R}$ are the discretized reflection coefficients. The form of the $5 \times 5$ submatrices shown in the upper right and lower left corners of Eq. (35) repeats down the diagonal. The other $5 \times 5$ submatrices given in Eq. (35) comprise the remainder of $\mathbf{R}$ and represent the interpolation terms.

With the boundary condition given in Eq. (34) the solution using discrete ordinates follows previous developments. ${ }^{6,7,11}$ Solution methods other than the one discussed here are possible, but in order to use the discrete ordinates method, an interpolation scheme was needed. One could imagine using a different discretization for the longitudinal and transverse intensities such that the mode conversion would occur directly from an $L$ ordinate to an $S V$ ordinate. This method may simplify the boundary condition but may create additional difficulties. Because these new ordinates would not necessarily be the Gaussian ordinates prescribed by the discrete ordinates method, more ordinates may be needed to obtain the necessary convergence. This option will not be examined further but merely noted as a possible alternative to the above interpolation scheme.

\section{STEADY-STATE RESULTS}

The polycrystalline URTE given in Eq. (3) was solved using the discrete ordinates method with the reflection boundary condition given in Eq. (5). An exponential twopoint correlation function was assumed such that $W(r)=e^{-\beta r}$ where $\beta$ is a measure of the inverse length scale which is on the order of the grain size. The excitation is a plane wave in the fluid with flux $F_{f}$ normally incident on the polycrystalline medium. The total flux entering the solid is then $T_{f L}^{\pi} F_{f}$, where $T_{f L}^{\pi}$ is the power transmission coefficient from the fluid to a longitudinal wave in the solid. For normal incidence $^{15}$

$$
T_{f L}^{\pi}=4 \frac{\rho_{f} c_{f}}{\rho c_{L}}\left(\frac{1}{1+\rho_{f} c_{f} / \rho c_{L}}\right)^{2} .
$$

The backscattered intensity in the fluid as a function of angle is shown in Fig. 4(a) for polycrystalline iron at low dimensionless frequency $x_{T}=\omega / c_{T} \beta=0.5$. The longitudinal and shear vertical contributions to the fluid intensity are shown separately in Fig. 4(b) to show the contribution from each mode. The critical angles for both wave types are apparent. The longitudinal contribution has a maximum at normal incidence and decreases until the longitudinal critical angle is reached. The shear vertical contribution is zero at normal incidence as expected and slowly increases until the longitudinal critical angle is reached. The shear vertical contribution then reaches a maximum and decreases until the shear vertical critical angle is reached. The angular dependence of the backscattered intensity for other frequencies is similar to Fig. 4 and is not shown. Figure 4 suggests that the fluid intensity will be rather constant within the longitudinal critical angle. Thus one may be able to avoid the large front face reflection by orienting the receiving transducer slightly away from normal for steady-state measurements.

The intensity in the solid as a function of dimensionless depth, $\tau=\kappa_{T} z$, is shown in Fig. 5 for polycrystalline iron at a high frequency $x_{T}=3.5$. The horizontal line is the $\mu=0$ line which denotes the demarcation between the upward

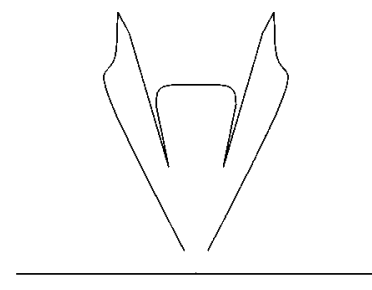

(a)

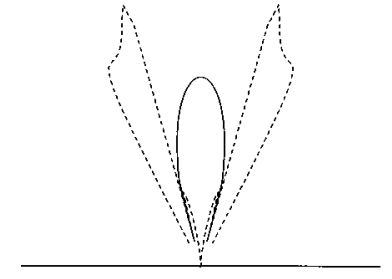

(b)
FIG. 4. Angular dependence of the steady-state intensity in the water as a function of angle for a normally incident longitudinal wave, $x_{T}=0.5$, without absorption: (a) total fluid intensity, (b) separation of longitudinal (solid line) and shear vertical (dashed line) contributions. 

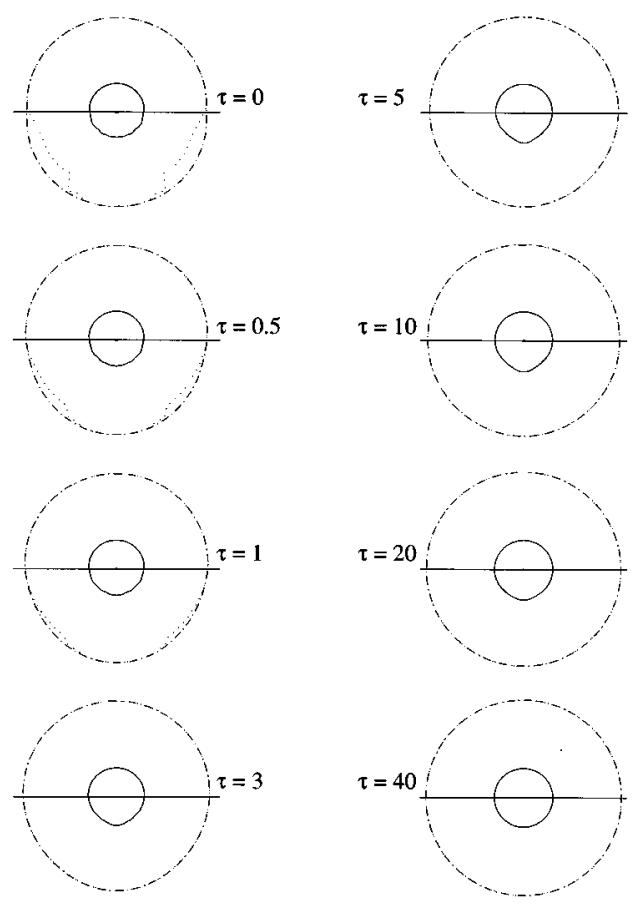

FIG. 5. Angular dependence of intensity as a function of dimensionless depth for iron in water, $x_{T}=3.5$, without absorption for the three modes $I_{L}$ (solid line), $I_{S V}$ (dotted), and $I_{S H}$ (dot dash).

$(\mu<0)$ and downward $(\mu>0)$ propagating intensities. The upward intensities at the surface, $\tau=0$, represent the intensities before they have passed through the interface. The upward intensities just beneath the surface are nearly isotropic. This result implies that the angular structure seen in Fig. 4 for the intensity in the fluid is almost entirely the result of the angular dependence of the transmission coefficients. The longitudinal and shear horizontal intensities in the downward direction are nearly isotropic as well. The shear vertical intensity has some structure which is the result of the angular dependence of the reflection coefficients. The shear horizontal intensity is symmetric about the $\mu=0$ line as expected.

Figure 5 may be compared with previous results for the same parameters with a transparent boundary condition. ${ }^{6}$ Away from the boundary, the vertical and horizontal shear intensities converge and become isotropic as before. With the boundary, the intensities reach the diffusive limit at much shallower depths. In fact, the shear intensities are isotropic after only three shear mean free paths. The longitudinal intensity takes longer to become isotropic as before, but is much more isotropic for all depths than previously. Thus it can be seen that the boundary reflection increases the approach to the diffusive limit. Results for lower frequencies are nearly isotropic at all depths.

The effect of the impedance mismatch between the fluid and solid is seen by comparing the above results for iron in water with results for a lesser mismatch aluminum-water system. Figure 6 contains the angular dependence as a function of angle for polycrystalline aluminum at $x_{T}=3.5$. The approach to the diffusive limit, especially for the longitudinal intensity, is much slower in this case and occurs at depths almost as deep as without the boundary effects.
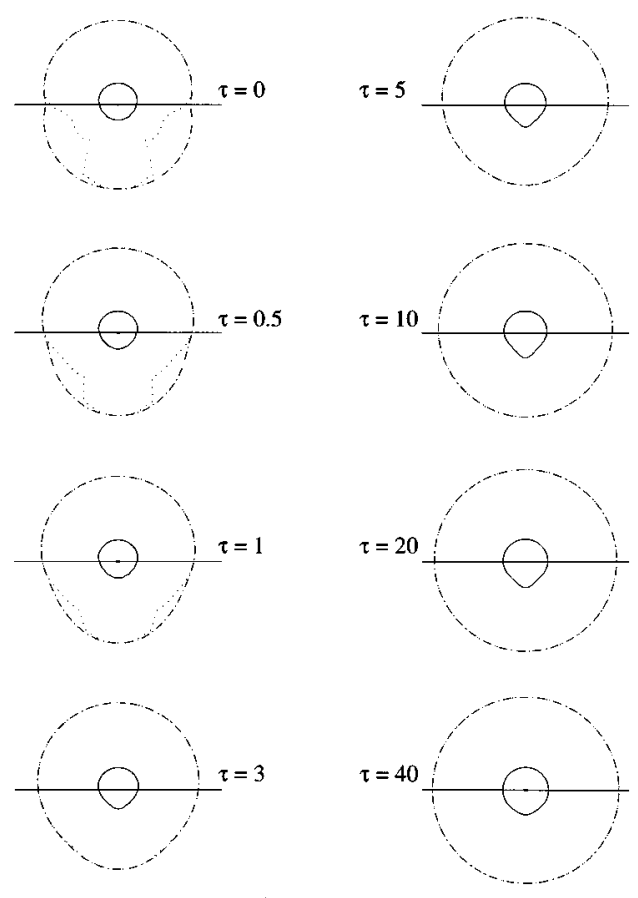

FIG. 6. Angular dependence of intensity as a function of dimensionless depth for aluminum in water, $x_{T}=3.5$, without absorption for the three modes $I_{L}$ (solid line), $I_{S V}$ (dotted), and $I_{S H}$ (dot dash).

The influence of the boundary on the steady-state multiply scattered solutions is intuitively satisfying. The diffusion regime is reached at shallower depths and is a function of the water-solid impedance mismatch. It was also shown that the upward intensity just beneath the surface is nearly isotropic and equipartitioned which may be a useful result for diffusivity measurement experiments.

\section{TIME-DEPENDENT RESULTS}

The time-dependent URTE was solved using the reflection boundary condition given above. Figure 7 shows the backscattered $(\mu=-1)$ fluid intensity as a function of dimensionless time, $\xi=c_{T} \kappa_{T} t$, measured in units of shear mean free times. This figure may be compared with results which did not include boundary effects. ${ }^{7}$ The peak in the multiply backscattered intensity with the boundary occurs later than before and is much wider. Both of these effects are the result of the energy having a more difficult time escaping the scattering medium. The effect of absorption is also more pronounced. The dimensionless absorption, $\tilde{v}_{T}=v_{T} / \kappa_{T}$, is measured with respect to the scattering attenuation. The absorption rate per wavelength was assumed constant such that $v_{L} c_{L}=v_{T} c_{T}$. The solutions with absorption deviate more from the zero absorption solution than the solutions without the boundary effects as expected. Because a typical ray is less likely to escape from the medium, it will be more greatly affected by absorption.

A comparison of the qualitative features of the two solutions may be examined by dividing the reflective solution by the two transmission factors $T_{L f}^{I}$ and $T_{f L}^{\pi}$. This comparison is shown in Fig. 8 for polycrystalline iron at $x_{T}=0.5$ without absorption. The two solutions agree at $\xi=0$ which 


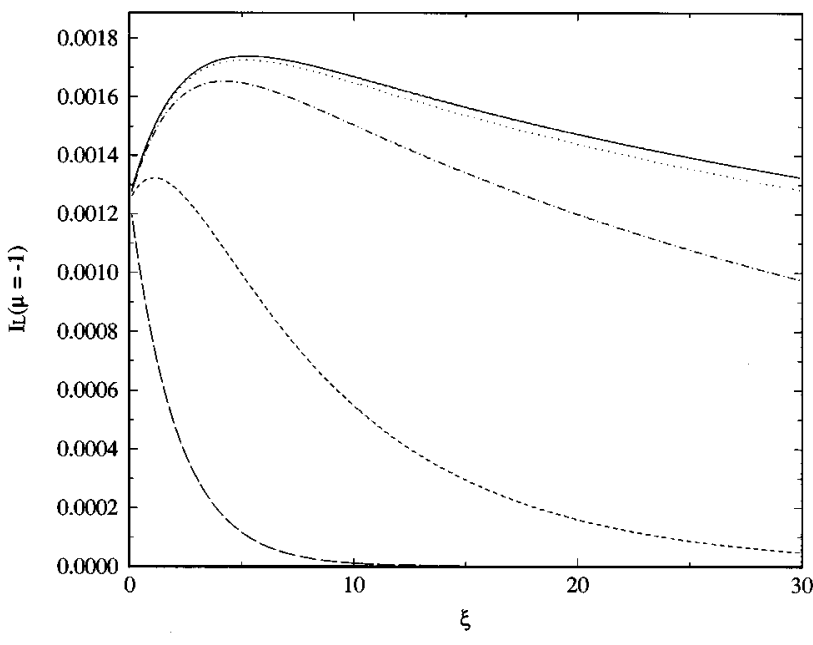

FIG. 7. Backscattered $(\mu=-1)$ fluid intensity versus dimensionless time, $\xi=c_{T} \kappa_{T} t$, at dimensionless inner frequency $x_{T}=0.5$ for different absorption rates: no absorption (solid line), $\tilde{v}_{T}=0.001$ (dotted), $\tilde{v}_{T}=0.01$ (dot dash), and $\tilde{v}_{T}=0.111$ (small dash). The singly scattered solution without absorption is shown by the large dashes.

provides confidence in the numerical work. The reflective solution is seen to rise much more quickly and decay much more slowly than the transparent boundary solution. The slower decay at late times is expected since the energy takes longer to escape the solid as discussed above. The quicker rate of increase was unexpected and not fully understood.

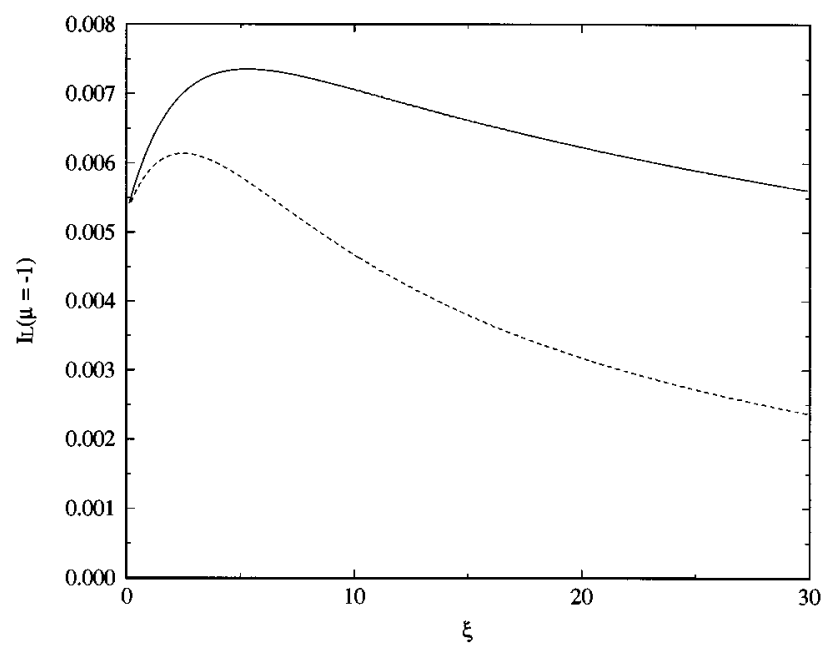

FIG. 8. Backscattered $(\mu=-1)$ fluid intensity versus dimensionless time, $\xi=c_{T} \kappa_{T} t$, at dimensionless inner frequency $x_{T}=0.5$ with (solid line) and without (small dash) the boundary. The solution with the boundary has been divided by the two transmission coefficients, $T_{L f}^{I}$ and $T_{f L}^{\pi}$.

\section{DISCUSSION}

A fluid-solid boundary condition has been presented for use with ultrasonic radiative transfer theory. This type of boundary condition is more realistic for comparison with experiments which are normally performed in a water bath. Both the steady-state and time-dependent results qualitatively behaved as expected. The steady-state solutions reached an isotropic, diffusive limit at shallower depths than with the transparent boundary and were nearly equipartitioned just beneath the specimen surface. The timedependent results decayed much more slowly and were more significantly affected by absorption. Both of these effects occur because the energy has a more difficult time escaping the scattering medium.

\section{ACKNOWLEDGMENT}

This work was sponsored by the National Science Foundation, Grant No. MSS-91-14360.

${ }^{1}$ F. J. Margetan, T. A. Gray, and R. B. Thompson, "A technique for quantitatively measuring microstructurally induced ultrasonic noise," Review of Progress in Quantitative NDE, edited by D. O. Thompson and D. E. Chimenti (Plenum, New York, 1991), Vol. 10, pp. 1721-1728.

${ }^{2}$ R. B. Thompson, F. J. Margetan, Y. H. K. Han, A. J. Paxson, and C. E. Shamblen, "Relationship of microstructure to backscattered ultrasonic noise," Review of Progress in Quantitative NDE, edited by D. O. Thompson and D. E. Chimenti (Plenum, New York, 1992), Vol. 11, pp. 16851691.

${ }^{3}$ M. D. Russell and S. P. Neal, "Grain noise power spectrum estimation for weak scattering polycrystalline materials using experimentally estimated backscatter coefficients: Normal incidence," Ultrasonics 32, 163-171 (1994).

${ }^{4}$ C. B. Guo, P. Holler, and K. Goebbels, "Scattering of ultrasonic waves in anisotropic polycrystalline metals," Acustica 59, 112-120 (1985).

${ }^{5}$ R. L. Weaver, "Diffusivity of ultrasound in polycrystals," J. Mech. Phys. Solids 38, 55-86 (1990).

${ }^{6}$ J. A. Turner and R. L. Weaver, "Radiative transfer and multiple scattering of diffuse ultrasound in polycrystalline media," J. Acoust. Soc. Am. 96, 3675-3683 (1994).

${ }^{7}$ J. A. Turner and R. L. Weaver, "Time dependence of multiply scattered diffuse ultrasound in polycrystalline media," J. Acoust. Soc. Am. 97, 2639-2644.

${ }^{8}$ S. Chandrasekhar, Radiative Transfer (Dover, New York, 1960).

${ }^{9}$ A. Ishimaru, Wave Propagation and Scattering in Random Media (Academic, New York, 1978), Vols. 1 and 2.

${ }^{10}$ L. Tsang, J. A. Kong, and R. T. Shin, Theory of Microwave Remote Sensing (Wiley, New York, 1985).

${ }^{11}$ J. A. Turner and R. L. Weaver, "Radiative transfer of ultrasound," J. Acoust. Soc. Am. 96, 3654-3674 (1994).

${ }^{12}$ J. D. Achenbach, Wave Propagation in Elastic Solids (Elsevier, New York, 1984).

${ }^{13}$ K. F. Graff, Wave Motion in Elastic Solids (Dover, New York, 1975).

${ }^{14} \mathrm{~J}$. Miklowitz, The Theory of Elastic Waves and Waveguides (NorthHolland, Amsterdam, 1978).

${ }^{15}$ L. M. Brekhovskikh and O. A. Godin, Acoustics of Layered Media I (Plane and Quasi-Plane Waves) (Springer-Verlag, Berlin, 1990).

${ }^{16} \mathrm{~K}$. Ergin, "Energy ratio of the seismic waves reflected and refracted at a rock-water boundary,” Bull. Seismol. Soc. Am. XX, 349-372 (1952). 